\title{
RELIABLE AND EFFICIENT DATA COMMUNICATION PROTOCOL FOR WBAN-BASED HEALTHCARE SYSTEMS
}

Nawel Yessad, Laboratoire d'Informatique Médical, Université de Bejaia, 06000 Bejaia, Algérie

Mawloud Omar*, Laboratoire d'Informatique Médical, Université de Bejaia, 06000 Bejaia, Algérie

\begin{abstract}
Wireless Body Area Networks (WBANs) become very attractive in the research community area and are getting growing interest because of their suitability for medical applications. They are designed such, they can be located on, in or around the patient body and are used to monitor medical signs and forward them to medical servers. Proficient energy and Quality of Service $(Q \circ S)$ are the main requirements for a dependable design in a such networks. In this paper, we propose a reliable and power efficient routing approach for healthcare systems with the aim to balance the trade-off between the QoS requirements and the energy consumption. Our approach operates in an efficient computation way, where the sink device manages the routing paths avoiding the sensors to be involved in computation and consequently on the energy consumption. We conducted intensive simulations and the obtained results show that our approach offers effective results in terms of transmission load, response time and energy consumption.
\end{abstract}

KEYWORDS: Healthcare, WBAN, Routing, Energy, QoS.

\section{INTRODUCTION}

With the increasing population around the world, as the elderly people who are more fragile to health diseases require a comprehensive healthcare system. The existing medical resources cannot satisfy future healthcare demands. Indeed, the healthcare resources are limited and it is impossible for most patients to afford long-term hospital stays due to economic restrictions, work, and even though their health status must be monitored in a real-time or short periodic time (Zhang, 2013). Further, the growing cost of healthcare has introduced great challenges for healthcare providers and healthcare industry. As results, there is great interest in using wireless monitoring medical systems to support remote patient monitoring in an unobtrusive, reliable and cost effective manner, thereby providing personalized sustainable services to the patients. For more details about the research effort in wireless communication standards for healthcare environments, kindly refer to (Rezaei, 2015) (Shanmugasundaram, 2016). In this context, Wireless Body Area Networks (WBANs) supporting 
healthcare applications are gaining worldwide interest for providing healthcare infrastructure and brings affordable and efficient healthcare solutions to people that would improve their quality of life. In a short time, WBANs represent the most widely emergent technologies that have the potential to significantly improve the e-health sector. WBANs are small networks, which consist of a number of lightweights, miniaturized sensors that could be located on the body as tiny intelligent patches, placed in or around the patient body in close proximity. These intelligent nodes monitor body functions and collect different physiological parameters like heart rate, glucose level, blood oxygen level, etc. (Ntouni, 2014). WBANs enable doctors and other medical staff to monitor continuously the health status of patients, individuals under medical risk, elders and chronically diseases people, people with disabilities, etc. WBANs need to stay connected to other networks by using other technologies in order to ensure that the patients data can reach the centre of treatment, while the subject is in a different place and the sensed data from the WBAN may ultimately be sent to a centralized healthcare repository for permanent records. Figure 1 illustrates the WBAN general architecture in the framework of the healthcare sector.

\section{Figure 1. WBAN general architecture for the healthcare sector}
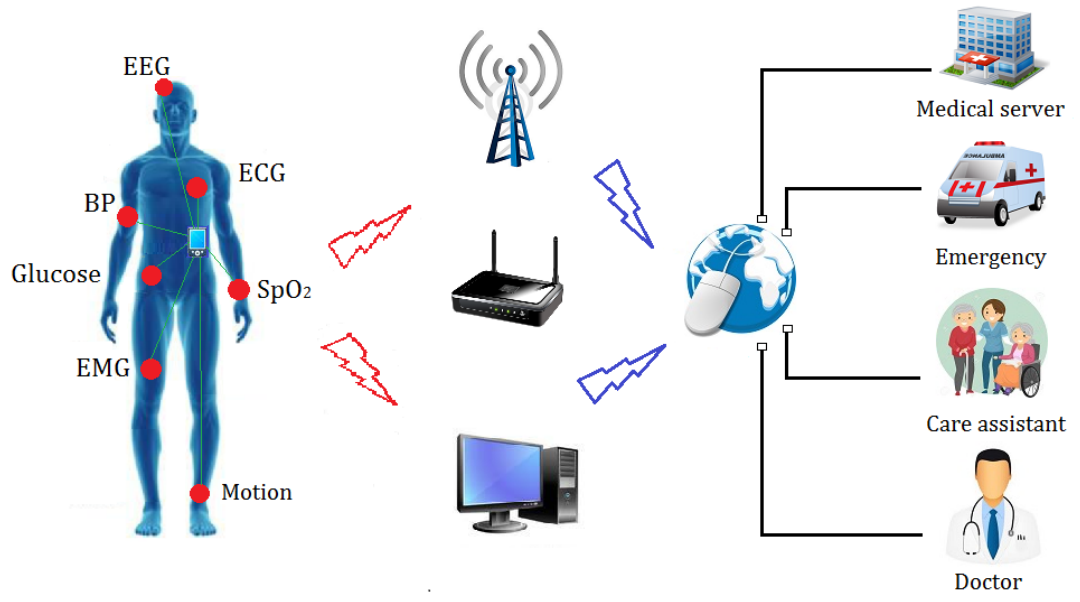

A crucial aspect in such networks is the communication among the sensor nodes to provide highly reliable communications for medical purposes. Routing is one of the challenging areas in WBANs research where developing efficient routing protocols in such networks is a non trivial task. First of all, this is due to the specific characteristics of WBANs. Secondly, the sensor nodes that form the network can be heterogeneous in terms of energy capacity, which is a major resource constraint to preserve. Furthermore, several medical applications require real-time data access, which presents a further constraint. Any routing approach designed for the healthcare purposes should be energy-efficient, increasing the network lifetime and QoS-aware to ensure reliable data communication. Although a research area is being done toward QoS routing 
protocols for WBANs taking the QoS requirement into account (Sharma, 2016), there still exist limitations. Few of these protocols consider energy awareness along with the QoS requirements. They consider that each sensor node is in charge to choose the best forwarder based on such parameters of its neighboring nodes, like the current energy state, location, velocity, etc. or based on a target objective function to relay the data packets. This view, being local and hence limited, may reduce the precision quality of the routing path.

In this paper, we propose a reliable and power efficient routing approach for WBAN-based healthcare systems. In our approach, the sensor nodes are completely discharged from the process of the best successor node selection. The sensor nodes are driven with routing paths, which are selected through a complete view of the WBAN topology based on the neighboring distances. The distance to reach the sink device has a great impact on many network metrics, namely the energy, the delay and the reliability. From the energy point of view, the consumption depends primarily on the transmission distance. If each sensor node transmits the data packet on longer distances to reach the sink device, the energy consumed over the communication may be very expensive. Otherwise, the energy consumption is reduced in the case of shortest transmission distances. As well as, the delay measurement depends also on the transmission distance and links failure can occur mainly in case of weak signal (longer distance). The reliability degree is affected and varies regarding the distance. The probability of the link failure is determined by the distance between two sensor nodes. However, sending packets over long distance relays reduces the reception cost, enough to avoid interference and the data packet error.

Our approach considers the distance as an important metric involved in the routing path selection. The sink device operates to get information about the different distances separating the network sensor nodes. Based on the latter, it creates and holds a global map of topology in order to determine the optimal route reaching any sensor node belonging to the network. The optimal routing path is selected depending on the data packet type generated by the sensor node, i.e., delay-sensitive, reliability-sensitive and regular packets. Our approach is very suitable for WBAN applications related to the healthcare sector. This is due to the fact that, it is energy-efficient, no complex computations involved and no extra operations are in charge to specific sensor nodes. This criterion is very important while balancing the load equally, and hence, balancing the energy consumption of sensor nodes.

The rest of the paper is organized as follows. We give, in Section 2, a state of the art on previous research effort in WBANs routing and we review some relevant and recent energy and QoS routing protocols in the framework of WBANs. In Section 3, we present the detailed description of our approach. In section 4, we present the simulation environment and discuss the obtained results. Finally, in Section 5, we conclude the paper. 


\section{PREVIOUS RESEARCH EFFORT}

In this section, we review and discuss some of the previous research works in the framework of WBAN routing.

The main challenge in WBAN routing is the development of energy and QoS aware approaches for reliable communication due to the sensitive application domains using this kind of network. Almost the existing solutions designed for wireless sensors and ad hoc networks (Balen, 2011; Panda, 2012) are often unsuitable for WBANs. Almost the applicable existing solutions are mostly based either on end-to-end routing with local decisions at each intermediate or an end-to-end routing path discovery and maintenance initiated by the source. However, energy and QoS design solutions based on new routing approaches still required. In this way, extensive research has been conducted and is still underway toward QoS requirements. In what follows, we discuss some relevant and recent energy and QoS routing protocols proposed in the framework of WBANs. In Table 1, we give an overall comparison of the reviewed QoS-based routing protocols.

Yousaf et al. (Yousaf, 2014) have proposed a thermal and energy aware routing protocol for WBANs. The proposed scheme supports body mobility and changes the route in case of hotspot detection. A hotspot is formed when a large volume of packets is sent from the sensor nodes of a small area, causing an obvious inconsistency in the network traffic.

Special type of sensor nodes known as relay nodes is used in (Elias, 2014) in order to minimize the energy consumption, to increase the lifetime and the reliability, as well as to decrease the thermal effects of the implanted biomedical sensor nodes. The relay nodes can be either well-placed on the human body or equipped within the clothing (wearable) and thus minimizing the small surgical operations, which are performed to replace the in-body biomedical sensor nodes.

Kaleem et al. (Kaleem, 2014) have proposed a routing protocol for WBANs based on a cost function, which is evaluated using the residual energy of sensor nodes and their distance from the sink to select the appropriate route. They have deployed two sensor nodes for an ECG and Glucose monitoring to forward their data directly to the sink device. Also, these two nodes cannot be selected as a parent because they monitor critical and important medical signs.

Sahndhu et al. (Sahndhu, 2015) have proposed a routing protocol for Balanced Energy Consumption (BEC) in WBANs. As well, the proposed solution is based on a cost function to select nearest forwarders to route the data to the sink. Furthermore, they have implemented a reactive routing protocol, where the sensor nodes send only critical data when their energy becomes less than a specific threshold. 
Adhikary et al. (Adhikary, 2016) have proposed a routing protocol for WBAN that uses sensor node information such as transmission power, velocity vector, residual energy and the current location to evaluate a cost function. They have added a fixed sensor node, doing so, a proper subset of relays and sensor nodes can be selected as forwarder based on the cost function value. Further, their protocol prevents formation of the hotspot.

Few routing protocols exist from the literature that consider the required QoS parameters based on the nature of data. In (Bangash, 2014; Bangash, 2015; Bangash, 2015), the authors have proposed a data-centric routing solutions for intra-WBANs. The proposed solutions ensure the best route selection considering the required QoS parameter based on the nature of sensory data and high path-loss of intra-WBANs. These requirements are considering while maintaining the temperature rise of the implanted bio-medical sensor nodes at a certain acceptable level. The data-centric routing solutions follow a modular architecture.

In (Khan, 2014; Razzaque, 2011; Djenouri \& Balasingham, 2009; Khan, 2015), the authors have presented an integrated QoS-aware routing protocols following a modular approach designed for inter-WBANs communication. They provide service differentiation by classifying the traffic into categories and determines an end-to-end route based on the traffic type. These solutions use separate modules for different types of data. Their main concern is to provide the required QoS based on the nature of data.

Monowar et al. (Monowar, 2014) have proposed a thermal-aware multiconstrained intra-body QoS-routing for WBAN (TMQoS) that integrates the QoS provisioning issue with thermal-aware in route selection for in-vivo sensor nodes. TMQoS selects alternative routing paths if hotspots are detected and uses a hotspot avoidance mechanism. It selects a shortest routing path from the source to destination meeting the QoS constraints, namely the delay and reliability. Later, Monowar et al. (Monowar, 2015) have designed a Thermalaware Localized QoS routing protocol for in-vivo sensor nodes in WBAN (TLQoS), that facilitates the system in achieving the desired QoS in terms of delay and reliability for diverse traffic types. It avoids the formation of highly heated nodes known as hotspots and keeps the temperature rise along the network to an acceptable level. TLQoS is based on modular architecture and implements potential-based localized routing that requires local neighborhood information.

Ahmad et al. (Ahmad, 2014) have proposed a Reliability Enhanced-Adaptive Threshold based Thermal-unaware Energy-efficient Multi-hop ProTocol (REATTEMPT), where positive features of both single-hop and multi-hop communication are used. Priority-based routing is implemented in the protocol 
for normal and critical data transmission. The routing paths are selected on the basis of the minimum hop count, which reduces the transmission delay.

Table 1. QoS based routing protocols in WBANs

\begin{tabular}{|c|c|c|c|c|c|c|}
\hline Protocol & $\begin{array}{c}\text { Energy } \\
\text { efficiency }\end{array}$ & $\begin{array}{c}\text { Reliable } \\
\text { delivery }\end{array}$ & Aggregation & $\begin{array}{c}\text { Modular } \\
\text { Architecture }\end{array}$ & $\begin{array}{c}\text { Traffic } \\
\text { differentiation }\end{array}$ & $\begin{array}{c}\text { Thermal } \\
\text { aware }\end{array}$ \\
\hline Yousaf, 2014 & Yes & Not-Supported & Yes & No & No & No \\
\hline Elias, 2014 & Yes & Not-Supported & No & No & No & No \\
\hline Kaleem, 2014 & Yes & $\begin{array}{c}\text { Direct } \\
\text { transmission }\end{array}$ & Yes & No & No & No \\
\hline Sahndhu, 2015 & Yes & Not-Supported & Yes & No & No & No \\
\hline Adhikary, 2016 & Yes & Not-Supported & Yes & No & No & Yes \\
\hline Bangash, 2014 & No & Probabilistic & No & No & Yes & Yes \\
\hline Bangash, 2015 & No & Probabilistic & No & Yes & Yes & Yes \\
\hline Bangash, 2015 & Yes & Probabilistic & No & Yes & Yes \\
\hline Khan, 2014 & Yes & Probabilistic & No & Yes & Yes & No \\
\hline Razzaque, 2011 & Yes & Probabilistic & No & Yes & Yes & No \\
\hline Djenouri, 2009 & Yes & Reception ratio & No & Yes & Yes & No \\
\hline Khan, 2015 & Yes & Probabilistic & No & Yes & Yes & No \\
\hline Monowar, 2014 & No & Probabilistic & No & Yes & Yes & Yes \\
\hline Monowar, 2015 & Yes & Loss ratio & No & Yes & Yes & Yes \\
\hline Ahmad, 2014 & Yes & Alternative & Yes & No & Yes & No \\
\hline
\end{tabular}

\section{OUR APPROACH}

In this section, we describe in detail the design of our approach. The key design goal is to achieve energy-efficient delivery of medical data while satisfying the QoS requirement.

\subsection{Problem Statement and General Ideas}

Efficient usage of sensor energy consumption, maximizing the network lifetime and QoS satisfaction are the main design considerations in WBANs. To design an efficient data communication protocol, we address directly the following challenges. It has been noted that most routing solutions, which have already been proposed, use an objective function in order to select the best neighbor based on the sensor node information such, location, velocity, energy residual, etc. The latter function is evaluated at each source node and at all the intermediate sensor nodes forwarding the data packet. This approach is local, reduces the precision quality of the selected routing path and may generate a considerable communication overhead in the maintenance of sensor nodes' states. To address this challenge, our key idea is to move much of the network 
and protocol complexity away from the sensor nodes -which are energyconstrained- into the sink device. With this manner, low energy consumption is involved, reduced communication overhead is generated, and low computation performed by the individual sensor nodes. A same routing path between a source node and the sink device may be used for several transmissions. However, the involved sensor nodes in the routing path can deplete rapidly their energy, and hence, the network lifetime may be compromised. In this context, we propose to distribute the routing functionality among all the network sensor nodes considering the energy consumption of each sensor node at each data transmission. For the QoS provisioning, we drive the traffic routing according to the QoS requirements to cater reliability maximizing and end-to-end packet delay minimizing.

\subsection{Network Model}

We consider a WBAN as an undirected graph $G=\langle\mathrm{V}, \mathrm{E}\rangle$, where $\mathrm{V}$ is the set of vertices representing the sensor nodes and $\mathrm{E}$ is the set of edges representing the communication links. We consider a deployment scenario, in which a set of $n$ sensor nodes $\mathrm{SN}_{\mathrm{i}}$ are attached to the human body measuring medical parameters, such as temperature, $\mathrm{ECG}$, blood pressure, $\mathrm{SpO} 2$ respiration, etc. They sense the data and transmit them to the sink device. The latter node is attached to the body center and it is responsible for collecting raw data and processing (coding, aggregation, etc.). To each sensor node is assigned a unique identifier before its deployment. We suppose that all the sensor nodes have the same maximal power range $\mathrm{R}$, where each one can control the transmission power in order to reach a particular neighbor node. The sink device is supposed to have relatively high energy and computing capabilities compared to the other sensor nodes.

There exists a link $E(i, j)$ between $S N_{i}$ and $S N_{j}$ if the distance $D_{(i, j)}$ between them is not larger than $R$, namely $D_{(i, j)} \leq R$. Undirected graph means bidirectional communication link. In other words, if $S N_{i}$ can be reached by the sensor node $S N_{j}$, then the latter can also be reached by $S N_{i}$. We denote the set of sensor nodes in the vicinity of $S N_{i}$ by $N_{S N_{i}}$ defined as $N_{i}=\left\{S N_{j}: D_{(i, j)} \leq R\right\}$. Although, there are many radio models in the literature, we use the most widespread one for the energy consumption (Heinzelman, 2000). Based on this model, the energy consumption cost for the transmission $E_{t}$ and reception $E_{r}$ of a $k$-bit data packet between two sensor nodes over a distance of $d$-meter are estimated in joules, respectively, by $E_{t}=\mathrm{k} \cdot\left(E_{\text {elec }}+E_{a m p} \cdot d^{2}\right)$ and $E_{r}=\mathrm{k} \cdot E_{\text {elec }}$. The parameters $E_{\text {elec }}$ and $E_{a m p}$ denote, respectively, the electronic energy and the transmitter amplifier.

Our approach for data routing takes in charge the individual QoS requirements, namely the reliability, the delay and the energy, which are the most important metrics for healthcare application scenarios. Our approach attempts to fulfil the 
required QoS metrics by selecting the best routing path based mainly on the transmission distances. Giving these requirements, we have three types of data packets: regular, reliability-sensitive and delay-sensitive packets. A regular packet is a kind of packet corresponding to regular measurements of patient medical parameters that typically indicate normal values and they do not have any specific requirement, such as the body temperature. A reliability-sensitive packet is a kind of packet, which should be delivered without loss, but not immediately or within a hard deadline, such as vital signs, respiration and $\mathrm{PH}$ monitors. A delay-sensitive packet is a kind of packet, which should be delivered in a short period of time.

\subsection{Overview}

We propose a destination-initiated efficient approach, which does not require sensor nodes to be involved in the routing path setup. The sensor nodes are discharged completely from the process of the best neighbor selection. Our approach exploits the high computation capability of the sink device to determine the optimal routing paths. The sink device holds a complete view of the WBAN topology including the different distances existing among the sensor nodes, thereby it determines the optimal routing path leading to any destination node in the network. This operation depends on the data packet type, i.e., regular, reliability-sensitive or delay-sensitive packet. The sink device can identify the QoS requirement in function of the sensor node identity, which specify the data packet type. The sink device balances the routing path selection to balance the energy consumption over each sensor node. The transmission energy consumption is balanced over the sensor nodes in order to prolong the network lifetime. Our approach consists of three phases, namely the topology discovery phase, the routing path determination phase, and the data routing phase. In what follows, we present the detailed description of each phase.

\subsection{Topology Discovery Phase}

The key operation in the discovery phase is the neighboring tables creation and their communication to the sink device in order to build-up the global view of the network topology. The sink device gets information to calculate and maintain the routing path used in the following steps. After the sensor nodes deployment, each node initiates the discovery protocol, which consists of broadcasting the hello packet. At the outset, a timeout is initialized and each sensor node $S N_{i}$ sends the packet using its maximal power of transmission. Upon receiving the packet, all the sensor nodes belonging to $N_{S N_{i}}$ use the signal strength of the received packet to estimate the distance. For this aim, RSSI (Received Signal Strength Indication) or LQI (Link Quality Indication) can be used (Mukhopadhyay, 2015). With this manner, each sensor node computes the distance separating it from each neighbor. A new entry is created for each 
Figure 2. Topology discovery phase

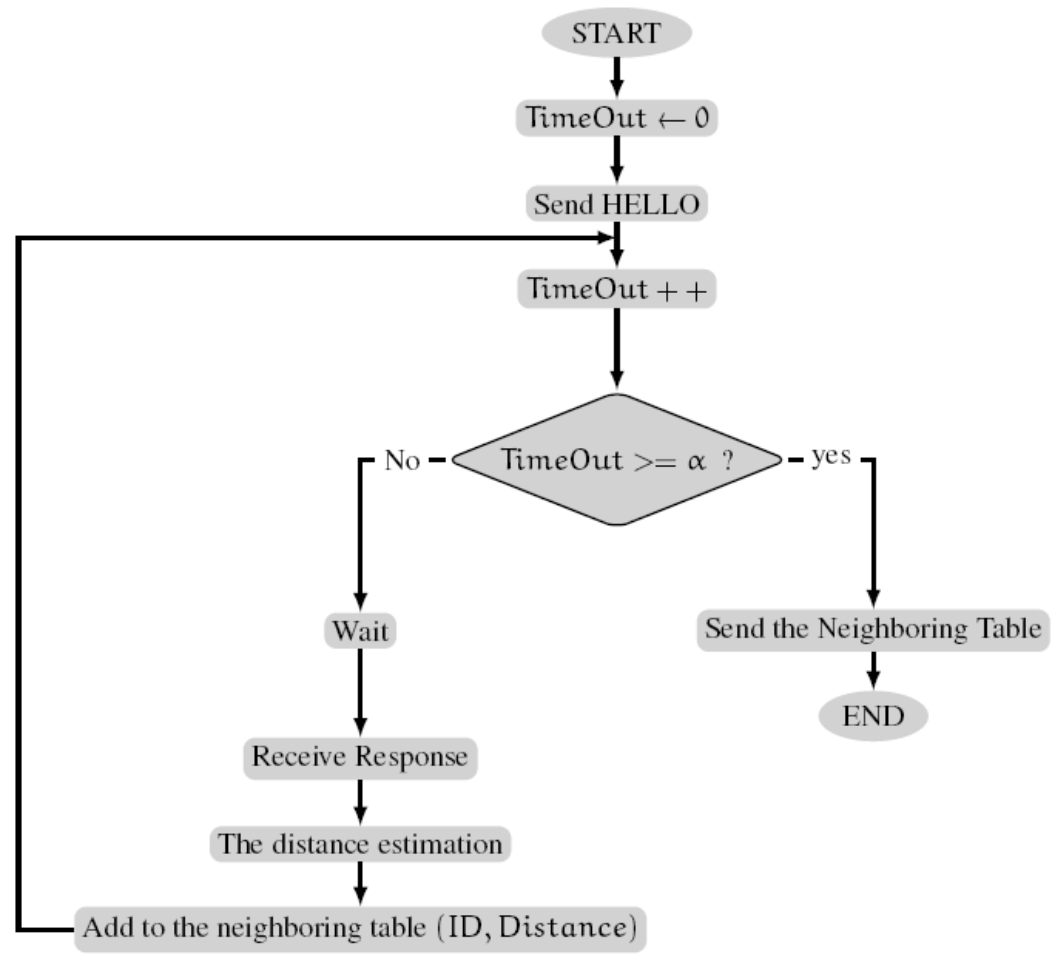

neighbor is created for each neighbor node, which includes two fields, namely the neighbor identifier and the separated distance. Otherwise, the timeout is incremented and compared to a threshold $\alpha$. If the timeout is greater than $\alpha$, then the process is achieved, and the neighboring tables are constructed then transmitted to the sink device, or else, the process is started again after the hello packet sending. Based on these tables, the sink device builds up locally the global view of the network topology. The process of this phase is illustrated in Figure 2 .

\subsection{Routing Path Determination Phase}

Upon the topology discovery is completed, the routing path determination phase starts and it is executed by the sink device in order to determine the optimal routing path leading to each sensor node $S N_{i}$ according to the sensed data packet type. The routing path is determined based on the global view of the network topology. The sink device explores from its local network topology the neighbor nodes progressively until reaching the targeted sensor node $S N_{i}$ while determining all the available routing paths leading to it. Regarding the data type, the sink device computes and identifies a single and optimal routing path 
based on the transmission distances of the intermediate sensor nodes. In the case of delay-sensitive packet, the best routing path $P^{*}$ is selected such $\operatorname{Dsum}\left(P^{*}\right)=\min \left(\operatorname{Dsum}\left(P_{1}\right), \operatorname{Dsum}\left(P_{2}\right), \cdots, \operatorname{Dsum}\left(P_{l}\right)\right)$, where $\operatorname{Dsum}(P)=$ $D_{(1,2)}+D_{(2,3)}+\cdots+D_{(|P|-1,|P|)}, P=S N_{1}\left\|S N_{2}\right\| \cdots \| S N_{|P|}$ and $l$ is the number of available routing paths. In the case of reliability-sensitive packet, the best routing path $P^{*}$ is selected such $D \max \left(P^{*}\right)=\min \left(\operatorname{Dmax}\left(P_{1}\right), \operatorname{Dmax}\left(P_{2}\right), \cdots\right.$ , $\left.D \max \left(P_{l}\right)\right)$, where $D \max (P)=\max \left(D_{(1,2)}, D_{(2,3)}, \cdots, D_{(|P|-1,|P|)}\right)$. In the case of the regular packet, the trade-off between the energy consumption and distance is maintained. Therefore, the best routing path $P^{*}$ is selected such $\operatorname{Emax}\left(P^{*}\right)=\min \left(\operatorname{Emax}\left(P_{1}\right), E \max \left(P_{2}\right), \cdots, E \max \left(P_{l}\right)\right)$, where $\operatorname{Emax}(P)=$ $\max \left(E_{1}, E_{2}, \cdots, E_{|P|}\right)$. Once this step is completed, the sink device builds-up and sends an advertisement packet $A d v$, which includes for each $S N_{i}$ an optimal routing path $P_{i}$ in order to transmit its future data packet. The format of the $A d v$ packet is structured as $A d v=\left\langle S N_{1}, P_{1}\right\rangle\left\|\left\langle S N_{2}, P_{2}\right\rangle\right\| \cdots \|\left\langle S N_{n}, P_{n}\right\rangle$. Upon receiving the data packet from a sensor node $S N_{i}$, the sink device reacts in order to update the preconfigured routing paths of all the sensor nodes. It performs again the exploration process, computes the routing paths and updates only the past used one in the routing process. Then, the sink device creates a new $A d v$ packet containing the previously computed and eventually the new-ones, and then sends the packet again. In short, we note that the sending of the determined routing path occurs only when the sink device receives a data packet from any sensor node. We denote with $\phi$ a no-updated routing path. Each $S N_{i}$ receives and typically checks its own packet field, which represents the insertion routing path field. If is targeted on the update, the new computed path is inserted, and hence, the sensor node caches this routing path for use in sending subsequent packets to the sink device. Otherwise, it is replaced by $\phi$ and the packet may be ignored. Let's consider an example of $A d v$ packet such as $A d v=\left\langle S N_{1}, \phi\right\rangle \|$ $\left\langle S N_{2}, N P_{2}\right\rangle\left\|\left\langle S N_{3}, \phi\right\rangle\right\| \cdots \|\left\langle S N_{n}, \phi\right\rangle$. A new routing path $N P_{2}$ is insered regarding the sensor node $S N_{2}$, and for the other nodes, there is no update for their routing paths. The process of this phase is illustrated in Figure 3.

\subsection{Data Routing Phase}

This phase is executed by a sensor node when a date has sensed and need to be communicated to the sink device. The sensor node $S N_{i}$ holds already its own routing path previously computed by the sink device. It embeds the complete routing path to the packet header. Let's consider a selected routing path $P=S N_{1}\left\|S N_{2}\right\| \cdots \| S N_{|P|}$, where the set of $S N_{i}$ is the intermediate sensor nodes and $S N_{|P|}$ represents the sink device. Upon receiving the data packet, each intermediate sensor node memorizes the identity of its predecessor node, checks its identifier from the list, and forwards the data packet to its successor. Again, this process is repeated until the packet reaches the targeted sensor node. The latter, upon receiving the packet, builds-up the replay Rep packet, which includes the medical data and returns it back to the sink device following the 
Figure 3. Routing path determination phase

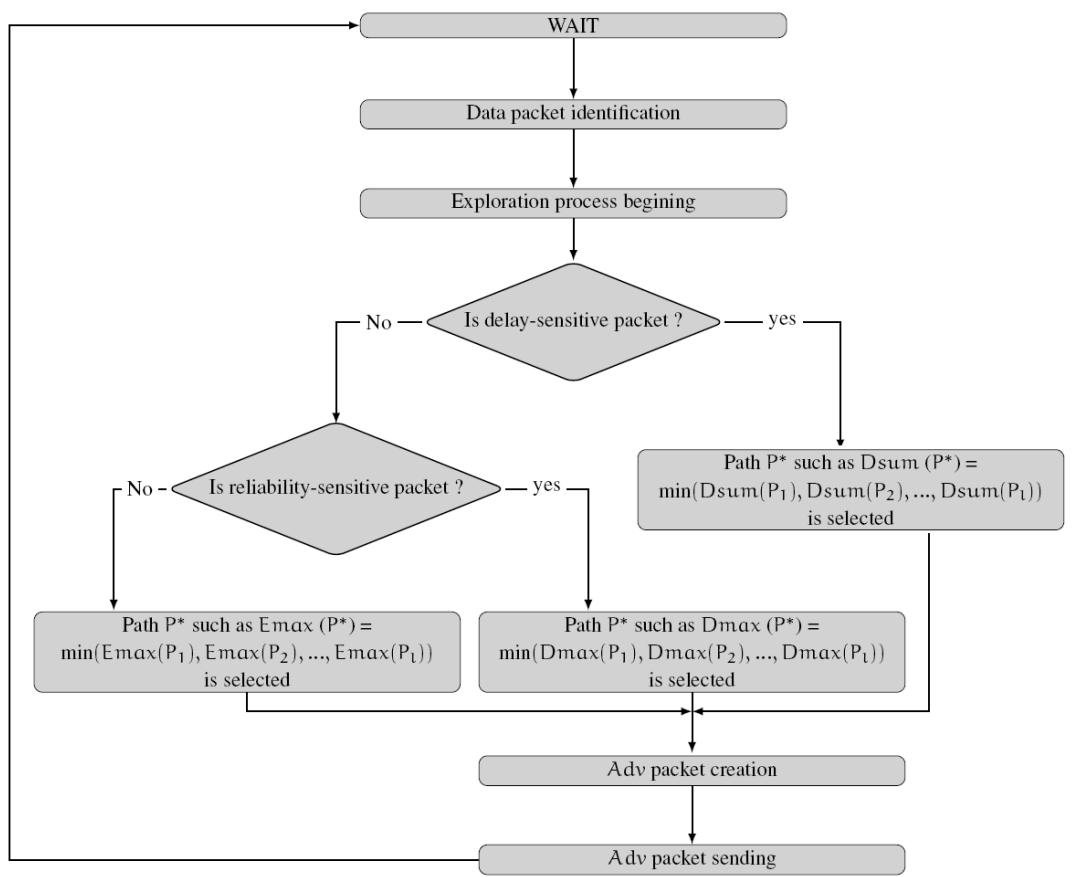

reverse route. Upon receiving the data packet, the sink device estimates the consumed energy of each $S N_{i}$ participated in the routing process according to the different transmission distances separating the intermediate sensor nodes on the basis of the energy model presented in Section 3.2. With this manner, the sink device balances the load among all the sensor nodes. The sink device considers that each intermediate sensor node in the selected routing path has transmitted two packets, namely $A d v$ and Rep. Therefore, it estimates and updates in its locally the consumed energy of the routing process for each sensor node $S N_{i}$ as $E_{i}=E_{i}+2 \cdot \mid$ packet size $\mid \cdot\left(E_{\text {elec }}+E_{\text {amp }} \cdot R^{2}\right)$.

\subsection{Inter-WBAN Data Communication}

In the first part we have addressed the intra WBAN routing, where we have presented a routing approach to handle and to manage the routing path selection. In this part, we address the inter-WBAN routing, where we consider an architecture, composed of a set of WABNs (clusters) supervised by a single base station, which is in charge to transmit the medical data to the remote medical server (cf. Figure 4). Each WBAN is considered as a cluster and the sink device as the cluster-head. The collected data of a given patient are delivered to the base station through a set of intermediate cluster-heads of other clusters using a multiple-hop communication. The point addressed in this phase is how to ensure a stable routing between the clusters in the aim to transmit the sensed data to the final destination. In this context, we have focused on the 
mobility parameter integration in the data communication process. We have incorporated a new technique of data communication based on the mobility estimation, which maintains stable routing paths between the clusters and the medical remote server. The mobility estimation is performed in function of the neighboring topology change and link availability. We introduce two metrics denoted by $I L$ and $O L$ representing, respectively, the number of in-links and out-links. These links could be or not available during an interval $[t, t+\Delta]$ between the clusters at each round. We were based on the estimation given in (Quwaider \& Biswas, 2009). At each round, each cluster-head $\mathrm{CH}_{i}$ computes the mobility index $M I_{i}(t)$ at the instant $t$ such as:

$$
M I_{i}(t)=\alpha \cdot \frac{O L_{i}(t)}{L_{i}(t-\phi)}+(1-\alpha) \cdot \frac{I L_{i}(t)}{L_{i}(t)}
$$

Where $L_{i}$ is the available link number and $\alpha$ is the mobility coefficient, which can be adjusted according to the application requirements. Afterwards, the cluster-head $\mathrm{CH}_{i}$ sends the computed index to the neighbors in order to be used in the stable neighboring selection process. Based on this concept, our aim is to select the most stable neighbor to forward the medical data. Finally, a clusterhead selects the neighbor with the minimum value of $M I_{i}(t)$. This process is repeated hop-by-hop until the data packet reaches the base station.

Figure 4. Inter-WBAN data communication

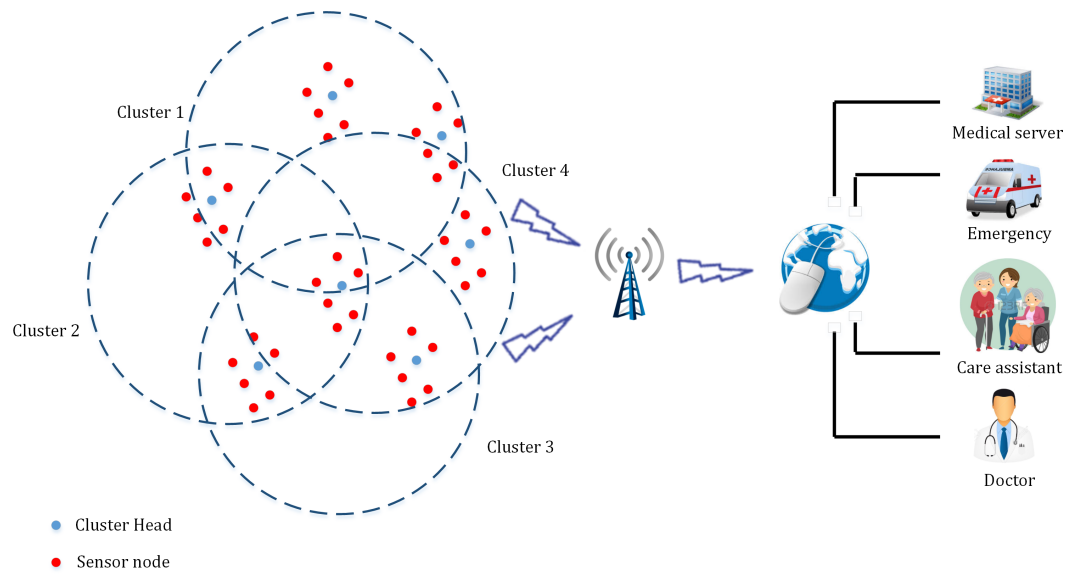

\section{PERFORMANCE EVALUATION}

The performance evaluation has been realized through simulations. We have compared our proposal to the conventional solution and the protocol TLQoS (Monowar, 2015). In this section, we present the simulator design, the performance metrics and finally we discuss the obtained results. 


\subsection{Our simulator design}

Network simulation methodology is widely used to verify the correctness, the performance and also to investigate the behavior of the evaluated protocols across different network topologies, different traffic, etc. Furthermore, it provides a controlled environment for researchers to perform protocol experimental evaluations before deployment when the actual hardware acquiring is difficult, infeasible, or impossible. Even for systems that may be impossible to physically realize, and allowing detailed inspection of interactions that may be too fast, or too complex to observe in a real system. There are previous efforts for designing simulation tools in the framework of wireless sensors and ad-hoc networks. Unfortunately, WBANs for healthcare applications present specific characteristics and constraints, which are not taken in charge with the existing simulators. In this context, we have developed a simulation tool for WBANs using Matlab environment. Figure 5 illustrates the detailed interaction process among the components, which we have implemented.

\subsection{Simulation Parameters}

The efficiency of our approach is investigated through extended simulation tests, which we have developed under Matlab/Simulink environment. We have simulated a WBAN through 8 sensor nodes supervised by a single sink device managing data packets of size $10 \mathrm{Kbit}$. We have considered a deployment area of $25 \mathrm{~m}^{2}$, where the set of sensor nodes is deployed in a deterministic manner at fixed positions and the sink device in the patient body center. Each sensor node is equipped with an energy source of $0.5 \mathrm{~J}$ and configured by a miniaturized wireless interface of a maximal power range of $0.5 \mathrm{~m}$. Table 2 summarizes the main simulation parameters.

Table 2. Simulation parameters

\begin{tabular}{|l|l|}
\hline Parameter & Value \\
\hline Deployment area of sensors & $25 \mathrm{~m}^{2}$ \\
\hline The coverage area of patients & $1000 \mathrm{~m}^{2}$ \\
\hline Deployment type & Determined \\
\hline Number of sensor nodes & 8 \\
\hline Number of sink & 1 \\
\hline Sink position & Patient body center \\
\hline Initial energy per sensor node & $0.5 \mathrm{~J}$ \\
\hline Data packet length & $10 \mathrm{Kbit}$ \\
\hline Maximal transmission range & $0.5 \mathrm{~m}$ \\
\hline Task type & Event driven \\
\hline Number of iterations & 100 \\
\hline
\end{tabular}

The aim of these experiments is to quantify the advantages of our approach. Hence, we were interested on three important performance metrics, namely the transmission load, the response time and the energy consumption. Through the 
transmission load, we analyse how well the proposed approach optimizes the communication overhead to gain in terms of efficiency. Moreover, reducing the transmission load allows to decrease considerably the negative impact of the generated radio signals from the sensors on the patient health.

Through the response time, we analyse the reactivity in order to promote the real-time criteria, which is highly required in emergency healthcare systems. Through the energy consumption, we evaluate the endurance of the WBAN in terms of lifetime. Prolonging the network lifetime decreases considerably the frequency of sensor relocation of the patient body. The energy consumption is computed from the total amount of energy consumed at the network over all the sensor nodes.

We have compared our routing approach to two protocols, namely the conventional approach and the protocol TLQoS:

- The conventional approach: the forwarding mechanism is based either on end-to-end routing with local decisions at each intermediate or an end-to-end routing path discovery and maintenance initiated by the source node. In both cases, the sensor nodes are in charge to choose the best next-hop (i.e., the forwarder) based on some parameters, like the current energy state, location, velocity, etc., or based on a target objective function. The latter function is evaluated at each source node and at all the intermediate sensor nodes forwarding the data packet.

- TLQoS (Thermal-aware Localized QoS routing protocol for in-vivo sensor nodes in WBAN) (Monowar, 2015): this approach achieves the desired QoS in terms of delay and reliability for various traffic types. TLQoS is based on different modules performing integrated operations in providing multiple QoS service with lower temperature rise. The proposed exploits a potentialbased localized routing approach in route selection that reflects its localized behavior requiring local neighborhood information. The forwarding mechanism is based on end-to-end routing with local decisions at each intermediate node.

\subsection{Obtained Results}

\subsubsection{Transmission Channel Reliability Impact}

In this subsection, we discuss the transmission channel reliability impact on the three performance metrics cited above.

Figure 6 plots the transmission load in function of the transmission channel reliability rate. Our approach achieves the best results compared to the conventional approach and TLQoS. With the latter solutions, the transmission load is still important. This is due to the broadcasted packets and exchanging between the sensor nodes in order to select the best next neighbor node, which has the required information to transmit the sensed data. Moreover, the reason 
Figure 5. Simulator design

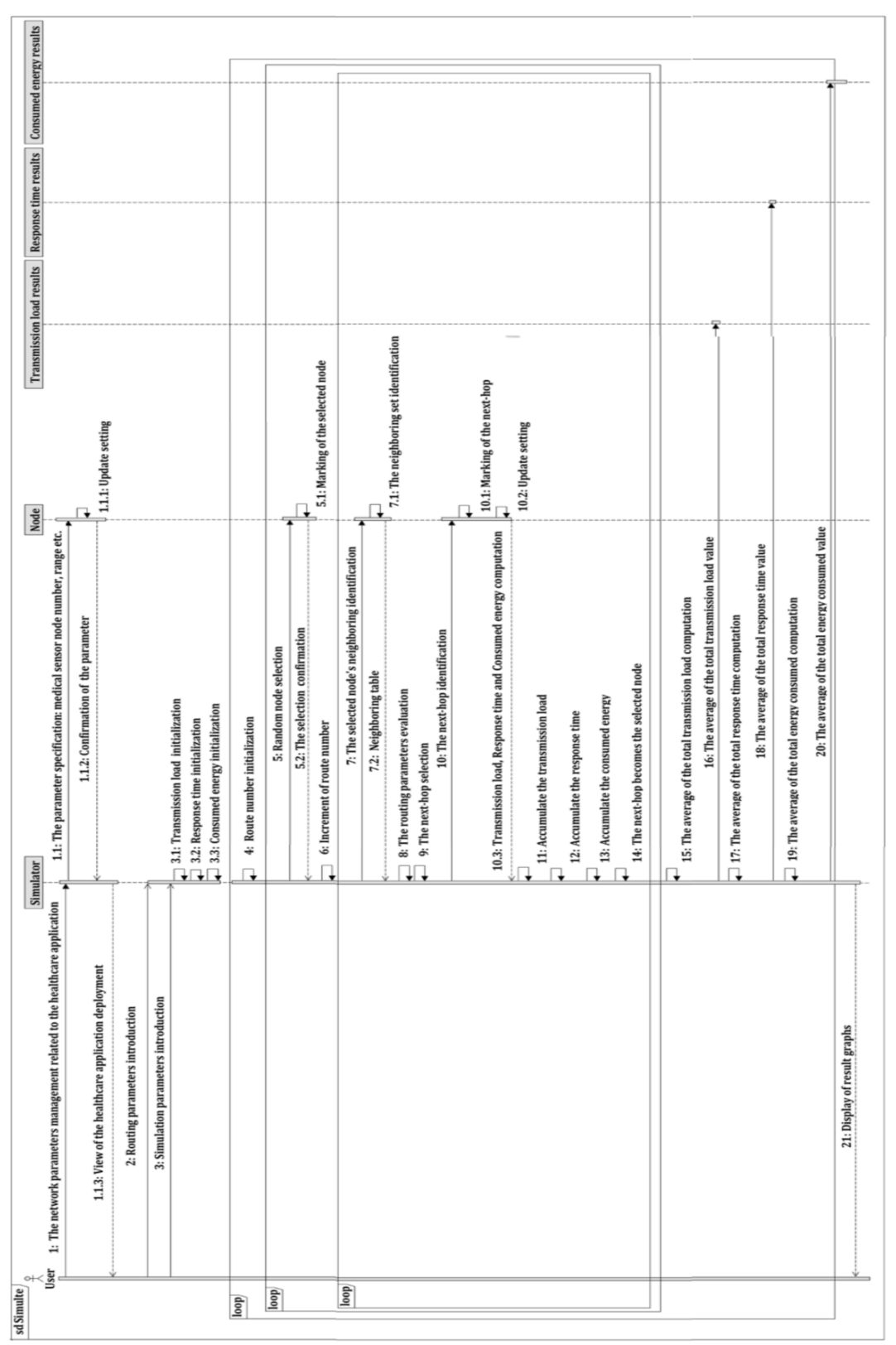


behind the low performances of TLQoS is the exchanging of parameters used in the selection process (location parameters). However, in our approach, the nodes are discharged from the selection process, therefore there are no additional exchanged packets. Unlike to the conventional approach, in addition to the measured data, sensor nodes require the exchange of several parameters, such as residual energy state, location, distance, load, etc.

Figure 6. Transmission load in function of transmission channel reliability

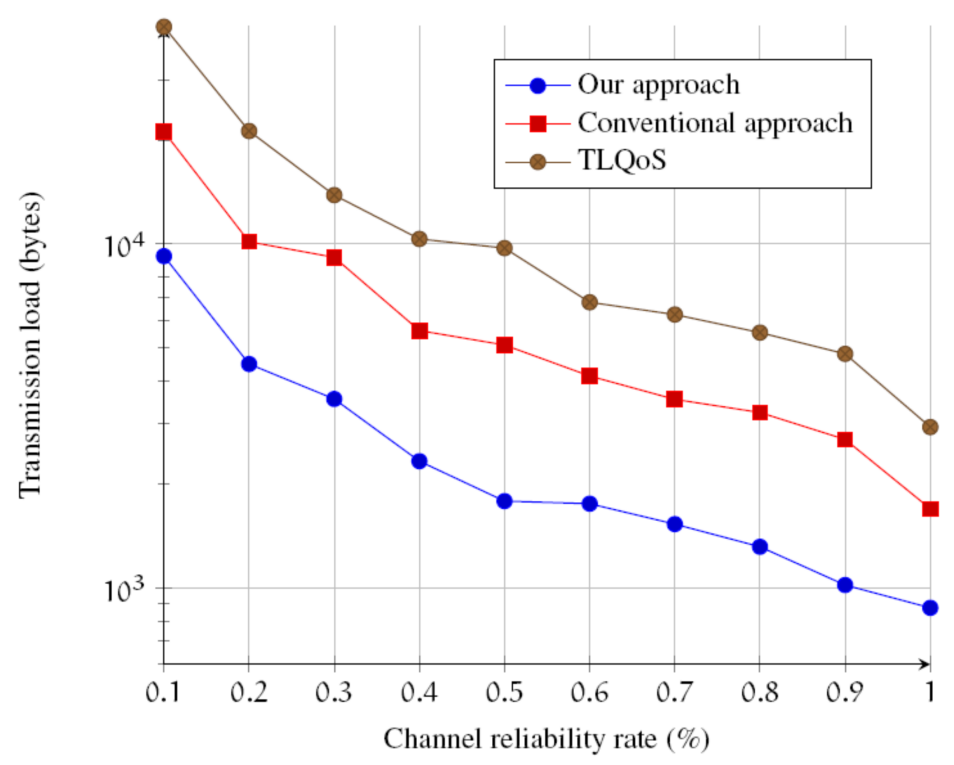

Figure 7 plots the response time in function of the transmission channel reliability rate. Regarding the results, our approach achieves again better results compared to the other protocols. In the case of the conventional approach and the TLQoS protocol, before the data is transmitted to the destination, a selection of the next neighbor node is performed, since this process will be repeated at each intermediate node, thus leading higher response time that continues to grow with the retransmissions in case of communication errors. Furthermore, TLQoS is based on a localization based QoS provisioning approach, which requires firstly the location parameters exchanged before the node forwarder selection. Therefore, the process leads to low performances in terms of response time. In our approach, the sensor nodes are driven by routes, which are selected by the sink node. We note that our approach offers a good effect in terms of network response time.

Figure 8 plots the energy consumption in function of the transmission channel reliability rate. Indeed, the results show that the energy consumed by our approach is lesser than the conventional approach and TLQoS. In this way, it 
allows an effective management of energy resources; therefore, the network lifetime is extended. The conventional approach and TLQoS don't take in charge the resource constraints of sensors. The sensor nodes compute the different functions to select the best neighboring, and hence consume more energy. On the other hand, our solution addresses these shortcomings by discharging the sensor node for the selection, where the sink device does the latter operation.

Figure 7. Response time in function of transmission channel reliability

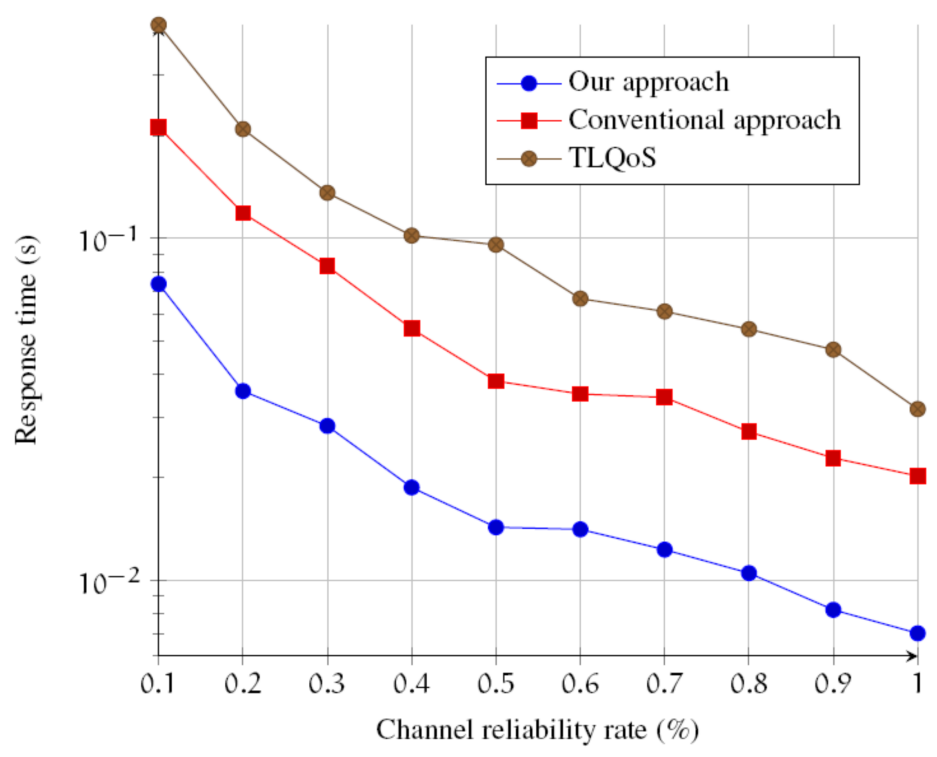

Figure 8. Energy consumption in function of transmission channel reliability

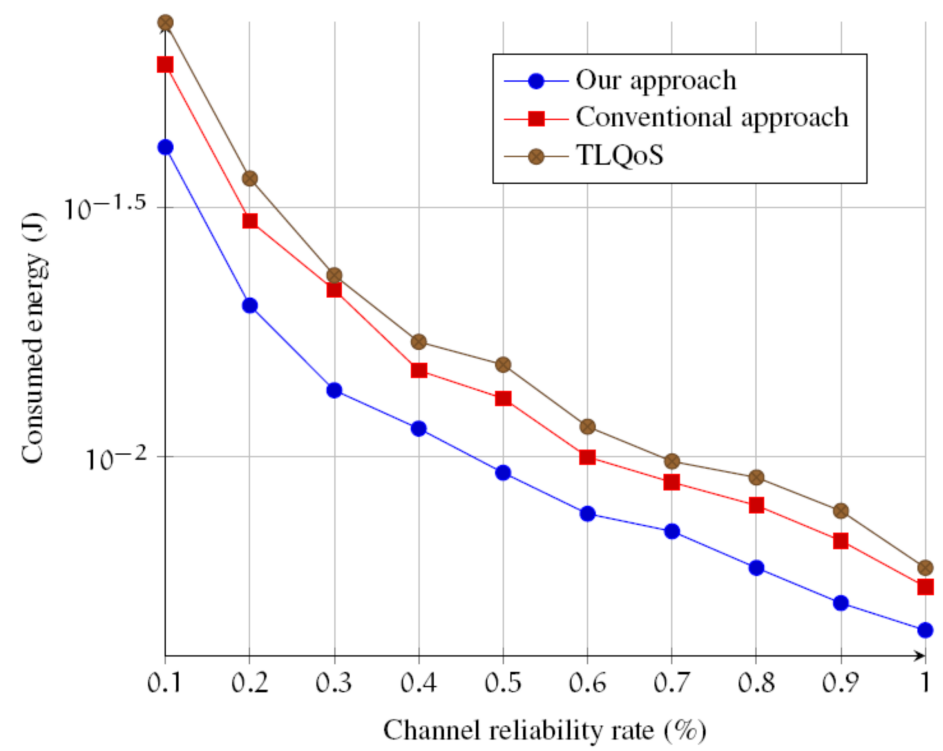




\subsubsection{Transmission Frequency Impact}

In this subsection, we discuss the impact of transmission frequency on the performances of the compared solutions.

Figure 9 plots the transmission load in function of the transmission frequency. Our approach demonstrates better results compared to the other approaches due to the transmission data amount, which represents just the sensed data. The other approaches show a considerable increase in load transmission due to the exchanged data amount among the sensor nodes necessary for path selection.

\section{Figure 9. Transmission load in function of transmission frequency}

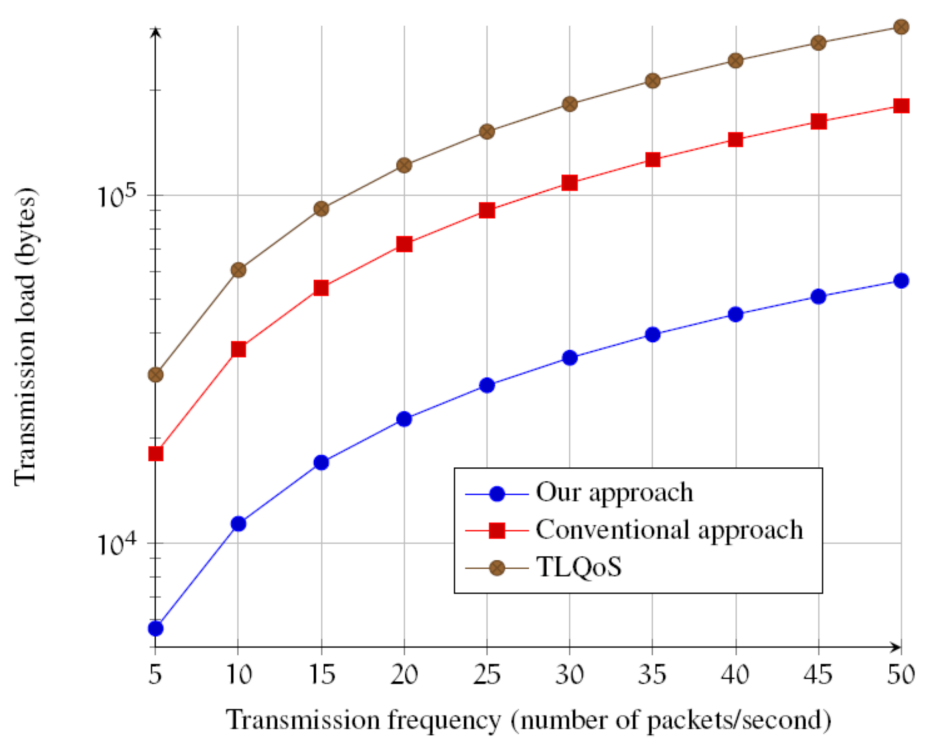

Figure 10 plots the response time in function of the transmission frequency. The obtained results show that our approach provides better performances compared to the conventional approach and TLQoS, with a tolerable increase where the exchanged packet number becomes very high. With the conventional approach, the response time is higher because of the packet number transmitted among the neighboring nodes before reaching the destination. In contrast, TLQoS implements the potential-based localized routing that requires the neighborhood information exchange.

Figure 11 plots the energy consumption in function of the transmission frequency. The consumed energy following our approach demonstrates the best performance compared to the other protocols. In the latter, the sensor nodes consume an important part of energy due to the transmission load. However, in our approach, the sensor nodes are pre-configured with routing paths which are 
selected and computed by the sink device, and hence, preserving the energy states of the other nodes. There are no additional packets transmitted between the neighboring nodes when routing regardless the data packet in the sensed event. Thus, our approach is suitable for the applications requiring emergency in the treatment and communication of medical data.

Figure 10. Response time in function of transmission frequency

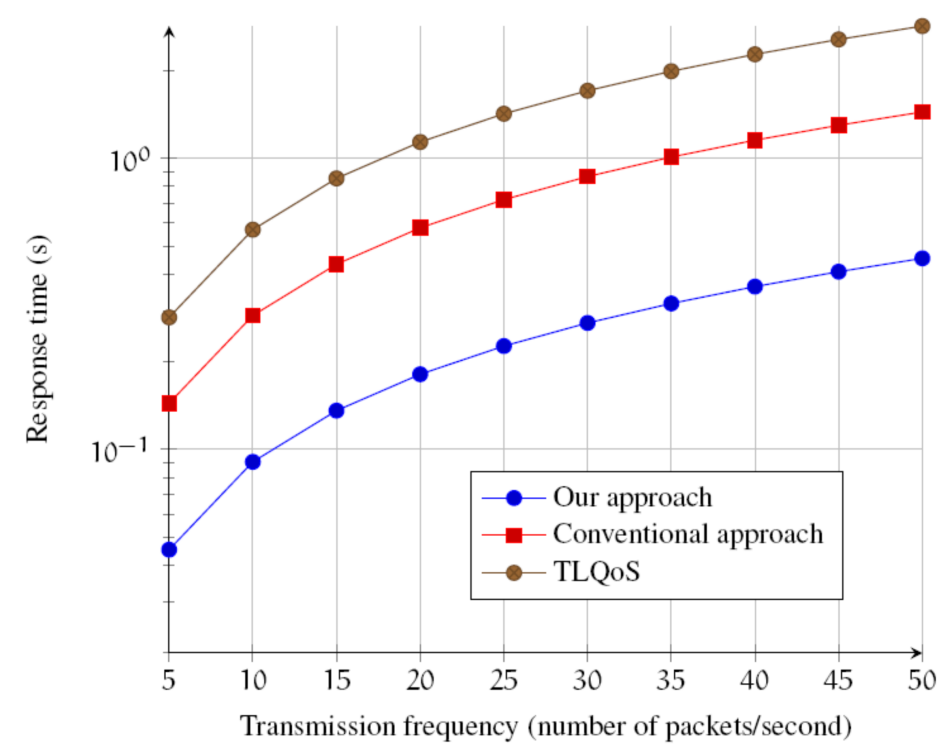

Figure 11. Energy consumption in function of transmission frequency

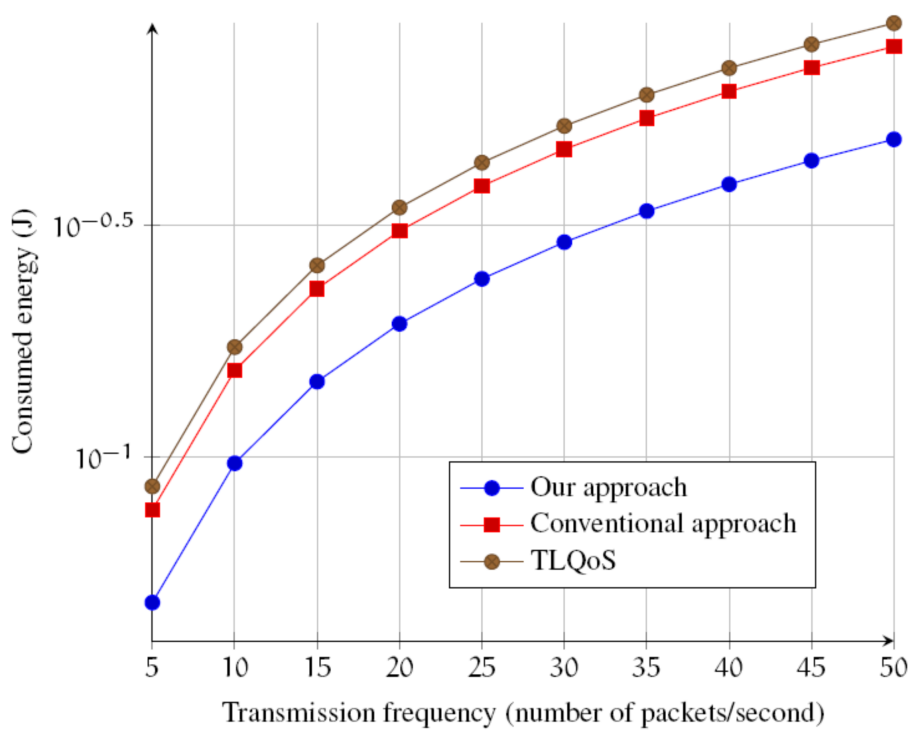




\section{CONCLUSION}

WBAN is one of the most promising technologies and emerging domains improving the quality of life by allowing remote patient monitoring and other ubiquitous health services. WBAN comprises tiny sensors placed on or inside the body. These sensors measure the patient vital information, and transfer them to the medical personnel for diagnosis without the patient displacement. In this type of networks, routing plays an important role due to the limited resources of sensor nodes. Transmission of medical data requires both energy and QoS aware approach in order to ensure efficient usage of the sensors resources and effective transmission of the data under certain situations. In this paper, we have proposed a destination-initiated efficient approach of routing, targeting the WBAN-based healthcare systems. The proposed approach charges the sink device in the best routing path selection without involving the other sensors. The optimal routing paths are determined according to the data packet type and based on the complete view of the WBAN topology. Our approach is energyefficient and QoS-aware as it balances the transmission energy consumption over all the sensor nodes. The effectiveness is evaluated through simulations, in which the obtained results are very encouraging.

\section{REFERENCES}

Sharma, R., Ryait, H-S. \& Gupta, A-K. (2016). Wireless Body Area Network: A Review. An International Journal of Engineering Sciences, 17 (1), 494-500.

Adhikary, S., Choudhury, S. \& Chattopadhyay, S. (2016). A new routing protocol for WBAN to enhance energy consumption and network lifetime. International Conference on Distributed Computing and Networking, 40-46.

Shanmugasundaram, G., Thiyagarajan, P \& Janaki, A. (2016). A survey of Cloud Based Healthcare Monitoring System for Hospital Management. International Conference on Data Engineering and Communication Technology, 549-557.

Rezaei, F., Hempel, M. \& Sharif, H. (2015). A Survey of Recent Trends in Wireless Communication Standards, Routing Protocols, and Energy Harvesting Techniques in E-Health Applications. International Journal of E-Health and Medical Communications, 6(1), 1-21.

Monowar, M-M., \& Bajaber, F. (2015). On Designing Thermal-Aware Localized QoS Routing Protocol for in-vivo Sensor Nodes in Wireless Body Area Networks. Sensors, 15(6), 14016-14044.

Sahndhu, M-M., Javaid, N., Imran, M., Guizani, M., Khan, Z-A. \& Qasim, U. (2015). BEC: A Novel Routing Protocol for Balanced Energy Consumption in Wireless Body Area Networks. International Wireless Communications and Mobile Computing Conference, 653-658. 
Bangash, J-I., Abdullah, A-H., Khan, A-W., Razzaque, M-A. \& Yusof, R. (2015). Critical data routing (CDR) for intra-wireless body sensor networks. Telecommunication, Computing, Electronics and Control, 13(1), 181-192.

Bangash, J-I., Khan, A-W., Abdullah, M-A. \& Yusof, R. (2015). Data-Centric Routing for Intra Wireless Body Sensor Networks. Journal of Medical Systems, 39(9), 91-103.

Khan, Z., Shyamala, C., Sivakumar, S., Phillips, W. \& Robertson, B. (2015). QPRR: QoS-Aware Peering Routing Protocol for Reliability Sensitive Data in Body Area Network Communication. The Computer Journal, 58(8), 17011716.

Mukhopadhyay, B., Sarangi, S. \& Kar, S. (2015). Performance Evaluation of Localization Techniques in Wireless Sensor Networks Using RSSI and LQI. National Conference on Communications, 1-6.

Kaleem, M. \& Mahapatra, R.P. (2014). Energy Consumption Using Network Stability And Multi-hop Protocol For Link Efficiency in Wireless Body Area Networks. Journal of Computer Engineering, 16(3), 113-120.

Ahmad, A., Javaid, N., Qasim, U., Ishfaq, M., Khan, Z-A. \& Alghamdi, T-A. (2014). RE-ATTEMPT: A new energy-efficient routing protocol for wireless body area sensor networks. International Journal of Distributed Sensors Networks, 2014, 1-9.

Khan, Z-A., Sivakumar, S., Phillips, W. \& Robertson, B. (2014). ZEQoS: A New Energy and QoS-Aware Routing Protocol for Communication of Sensor Devices in Healthcare System. International Journal of Distributed Sensors Networks, 2014, 1-18.

Monowar, M-M., Hassan, M-M, Bajaber, F., Hamid, M-A. \& Alamri, A. (2014). Thermal-Aware Multiconstrained Intra body QoS Routing for Wireless Body Area Networks. International Journal of Distributed Sensor Networks, 2014, 1-14.

Elias, J. (2014). Optimal design of energy-efficient and cost-effective wireless body area networks. Ad Hoc Networks, 13 (B), 560-574.

Ntouni, G-D., Lioumpas, A-S. \& Nikita, K-S. (2014). Reliable and Energy Efficient Communications for Wireless Biomedical Implant Systems. IEEE journal of biomedical and health informatics, 18(6), 1848-1856.

Bangash, J-I., Abdullah, A-H., Razzaque, M-A., Guizani, M. \& Khan, A-W. (2014). Reliability aware routing for intra-wireless body sensor networks. International Journal of Distributed Sensors Networks, 2014, 1-10.

Yousaf, S., Ahmed, S., Akbar, M., Javaid, N., Khan, Z.A. \& Qasim, U. (2014). Co-CEStat: Cooperative critical data transmission in emergency for static wireless body area network. Ninth International Conference on Broadband and Wireless Computing, Communication and Applications, 127-132. 
Zhang, S., Qin, Y-P., Mak, P-U., Pun, S-H. \& Vai, M-I. (2013). Real-time medical monitoring system design based on intra-body communication. Journal of Theoretical and Applied Information Technology, 47(2), 649-652.

Panda, I. (2012). A Survey on Routing Protocols of MANETs by Using QoS Metrics. International Journal of Advanced Research in Computer Science and Software Engineering, 2(10), 120-129.

Razzaque, M-A., Hong, C-S. \& Lee, S. (2011). Data-centric multiobjective QoS-aware routing protocol for body sensor networks. Sensors, 11(1), 917937.

Balen, J., Zagar, D. \& Martinovic, G. (2011). Quality of Service in Wireless Sensor Networks: A Survey and Related Patents. Recent Patents on Computer Science 2011, 4(3), 188-202.

Djenouri, D. \& Balasingham, I. (2009). New QoS and geographical routing in wireless biomedical sensor networks. In Proceedings of the 6th International Conference on Broadband Communications, Networks and Systems, 1-8.

Quwaider, M. \& Biswas, S. (2009). Probabilistic Routing in On-body Sensor Networks with Postural Disconnections. In Proceeding of the 7th ACM international symposium on Mobility management and wireless access, 149158.

Heinzelman, W-R., Chandrakasan, A. \& Balakrishnan, H. (2000). Energyefficient communication protocol for wireless microsensor networks. In Proceedings of the Hawaii international conference on system sciences. 